\title{
Antibacterial Resistance and Molecular Typing of Methicillin-Resistant Staphylococcus aureus in a Kuwaiti General Hospital
}

\author{
E.E. Udo ${ }^{\text {a }}$ N. Al-Sweih ${ }^{\text {a }}$ S. Mohanakrishnan ${ }^{\text {a }}$ P.W.J. West ${ }^{\text {b }}$ \\ ${ }^{a}$ Department of Microbiology, Faculty of Medicine, Kuwait University, and ${ }^{b}$ Department of Medical Laboratory \\ Technology, Faculty of Allied Health Sciences, Kuwait University, Safat, Kuwait
}

\begin{abstract}
Key Words
Methicillin-resistant Staphylococcus aureus · Fusidic acid resistance Mupirocin resistance $\cdot$ Pulsed-field gel electrophoresis
\end{abstract}

\begin{abstract}
Objective: To investigate antibiotic resistance and genetic relatedness of methicillin-resistant Staphylococcus aureus (MRSA) isolated in a general hospital in Kuwait over a period from 1996 to 1998 and 2001. Material and Methods: The isolates were characterized by antibacterial susceptibility testing, coagulase serotyping, coagulase gene polymorphism (coag-RFLP) and pulsed-field gel electrophoresis (PFGE). Results: The MRSA isolates were highly resistant to gentamicin, kanamycin, ciprofloxacin, tetracycline, fusidic acid and mupirocin. The prevalence of gentamicin, kanamycin, streptomycin, tetracycline and erythromycin resistance remained high (80-96\%) throughout the study period, but the prevalence of resistance to ciprofloxacin, fusidic acid and mupirocin steadily increased. The already high mupirocin resistance level increased from 12.5 in 1996, to $85.7 \%$ in 2001, and the fusidic acid resistance varied between 70.8 and $85.7 \%$. In contrast, chloramphenicol and trimethoprim resistance declined from 25 and $29 \%$ in 1996 to 4.7 and $14.2 \%$ in 2001 , respectively. The majority (91.5\%) of the isolates were coagulase serotype 4 . Alul restriction endonuclease analysis of amplified coagulase gene gen-
\end{abstract}

erated four coag-RFLP patterns: $92 \%$ of them were coagRFLP type 1, while types 2,3 and 4 were $3.5,4.6$ and $1.1 \%$ respectively. PFGE differentiated them into seven pulsotypes (PFGE types $1-7)$. The PFGE type 1 pulsotype constituted $90.2 \%$ of the isolates. Isolates with the type $A$ coag-RFLP also had the type1 PFGE pulsotypes. Conclusion: The concordant results of PFGE and coag-RFLP demonstrated the presence of a persistent MRSA clone in the hospital during the study period.

Copyright (C) 2006 S. Karger AG, Basel

\section{Introduction}

Methicillin-resistant Staphylococcus aureus (MRSA) continues to be an important cause of nosocomial and community-acquired infections worldwide. MRSA, especially those associated with the healthcare environment, often have multiple resistance to antibacterial agents, including resistance to the aminoglycosides, macrolides, tetracyclines, fluroquinolones, chloramphenicol, heavy metals and antiseptics. Some MRSA strains can colonize patients, healthcare workers and the environment, and are very difficult to eradicate once they become endemic in healthcare facilities $[1,2]$. MRSA can be transmitted between hospitals in a city [3, 4] and across international boundaries [5-7] as a result of human movements. Therefore, controlling their spread requires effective surveillance measures that can provide sound, reliable and long-

\section{KARGER}

Fax +4161306 1234

E-Mail karger@karger.ch

www.karger.com
(C) 2006 S. Karger AG, Basel

$1011-7571 / 06 / 0151-0039 \$ 23.50 / 0$

Accessible online at:

www.karger.com/mpp
Dr. Edet E. Udo

Department of Microbiology, Faculty of Medicine

Kuwait University, P.O. Box 24923

Safat 13110 (Kuwait)

Fax+965 533 2719, E-Mail edet@hsc.edu.kw 
term profile of the emergence of new resistance to existing antibacterial agents as well as the evolution and spread of new resistant clones [8]. Surveillance data on antimicrobial resistance are important for every institution, for monitoring the emergence and spread of these resistant organisms [9].

In Kuwait, MRSA isolated from patients in a teaching hospital [10] and a burns unit [11, 12] have been characterized. However, information on MRSA isolated from general hospitals in the country is lacking. The prevalence and clonal types of MRSA may vary considerably among hospitals in the same city or country $[4,6]$. Therefore, there is a need to study the characteristics of MRSA strains obtained from patients in general hospitals in Kuwait, for their antibacterial resistance patterns and clonal composition. In this study, MRSA obtained from patients in a Kuwait general hospital in 1996-1998 and 2001 were studied for their susceptibility to antibacterial agents, and typed using phenotypic and genotypic methods to assess changes in their resistance patterns and clonal types.

\section{Materials and Methods}

\section{Hospital}

The Farwania hospital is a 350-bed general hospital with Obstetrics and Gynecology, Pediatrics, Medical and Surgical wards, an Intensive Care Unit and an Outpatient Department (OPD). It serves a population of approximately 150,000 people of different nationalities. The Farwania Hospital was selected for this study because MRSA isolated from it had been preserved from 1996 onwards with the exception of 1999, and were available for this study.

\section{MRSA Isolates}

The MRSA isolates were obtained from routine clinical samples of 88 patients, in all the wards and the OPD at the Farwania Hospital, in 1996: 24; 1997: 25; 1998: 18, and 2001: 21. No MRSA was isolated in the obstetrics and gynecology wards during the study period. Eleven of the isolates were from patients visiting the OPD. The medical ward was the major source of samples, yielding 44 samples. The orthopedic, surgical, ICU and pediatric wards contributed $11,8,7$ and 6 MRSA isolates, respectively.

The isolates were identified by characteristic growth patterns by culturing on mannitol salt agar and Gram's stain, and positive tube coagulase test. Isolates were preserved at $-80^{\circ} \mathrm{C}$ in $20 \%$ (v/v) glycerol and subcultured in Brain heart infusion broth and Brain heart infusion agar.

\section{Antibiotic Susceptibility Testing}

Susceptibility testing was performed by the disk diffusion method on Mueller Hinton agar as described previously [13]. Susceptibility testing for methicillin was performed on Mueller Hinton agar plates with disk containing $5 \mu \mathrm{g}$ of methicillin and incubated at $30^{\circ} \mathrm{C}$ for $24 \mathrm{~h}$. Methicillin resistance was confirmed by detecting $\mathrm{PBP} 2 \mathrm{a}$ in culture supernatants, using a rapid latex agglutination kit according to the manufacturer's instruction (Denka-Seiken, Japan). For susceptibility to heavy metals and nucleic acid-binding compounds, 6-mm disks (Whatman AA disks) impregnated with cadmium acetate $(130 \mu \mathrm{g})$, propamidine isethionate $(50 \mu \mathrm{g})$ and ethidium bromide $(60 \mu \mathrm{g})$ were used. Minimum inhibitory concentrations of mupirocin, vancomycin and teicoplanin were determined with E-test strips as per manufacturer's instructions (AB Biodisk, Solna, Sweden). S. aureus strain ATCC25923 was used for quality control of susceptibility testing. $\beta$-Lactamase production was tested with nitrocefin, by following the manufacturer's instructions (Oxoid, Basingstoke, UK). S. aureus strain ATCC29213 was used as a positive control.

\section{Strain Typing}

The coagulase serotypes were determined with the staphylococcal coagulase antisera according to the manufacturer's instructions (Denka-Seiken, Japan). The coagulase gene was amplified using published primers [14]. The nucleotide sequence $\left(5^{\prime} \rightarrow 3^{\prime}\right)$ of standard primers purchased were COAG-2: ACC ACA AGG TAC TGA ATC AAC G and COAG-3: TGC TTT CGA TTG TTC GAT GC (Gibco BRL, Paisley, UK). The amplified products were digested with $5 \mathrm{U}$ of $A l u \mathrm{I}$ restriction enzyme, incubated at $37^{\circ} \mathrm{C}$ and separated by agarose gel electrophoresis $(2.5 \%$ agarose, $w / v)$ in $1 \times \mathrm{TBE}$ buffer for $2.5 \mathrm{~h}$ at $85 \mathrm{~V}$. The gel was stained with ethidium bromide $(1 \mathrm{mg} / \mathrm{ml})$ and photographed under UV illumination. S. aureus strain ATCC25923 and Staphylococcus epidermidis strain ATCC12228 were used as positive and negative controls, respectively. For pulsed-field gel electrophoresis (PFGE), cells were prepared in agarose blocks (PFGE grade, BioRad Laboratories, Hercules, Calif., USA) and digested with SmaI as described previously [11]. Interpretation of PFGE patterns of all of the 88 isolates was made, using the recommendation by Tenover et al. [15]. Isolates that differed by one to five bands were classified as related, and those that differed by six or more bands were considered to be unrelated.

\section{Genetic Location of Resistance Determinants}

Plasmids were isolated by the cetyltrimethyl ammonium bromide method, separated by agarose gel electrophoresis and sized as described previously [11]. Loss of resistance and plasmids was investigated by curing at $43^{\circ} \mathrm{C}$ [11]. Plasmids were transferred in mixed-culture transfer and conjugation experiments carried out as described previously [16].

\section{Results}

\section{Antibiotic Susceptibility Patterns}

All 88 isolates were methicillin resistant. This was confirmed by the production of PBP2a, and the fact that all the test samples produced penicillinase. All the isolates were susceptible to linezolid, teicoplanin and vancomycin, with none expressing intermediate glycopeptides resistance. The minimum inhibitory concentration values of vancomycin and teicoplanin were $0.5-3.0 \mathrm{mg} / \mathrm{l}$.

The prevalence of antibacterial resistance is summarized in table 1. A majority of the isolates were resistant 
Table 1. Antibiotic resistance trends of MRSA isolates

\begin{tabular}{lcccc}
\hline Antibiotics & \multicolumn{4}{l}{ Resistance prevalence } \\
\cline { 2 - 5 } & $\begin{array}{l}1996 \\
(\mathrm{n}=24)\end{array}$ & $\begin{array}{l}1997 \\
(\mathrm{n}=25)\end{array}$ & $\begin{array}{l}1998 \\
(\mathrm{n}=18)\end{array}$ & $\begin{array}{l}2001 \\
(\mathrm{n}=21)\end{array}$ \\
\hline Gentamicin & $22(91.7)$ & $24(96.0)$ & $15(83.3)$ & $17(81)$ \\
Kanamycin & $22(91.7)$ & $25(100.0)$ & $18(100)$ & $18(85.7)$ \\
Streptomycin & $20(83.3)$ & $24(96.0)$ & $14(78)$ & $16(76.2)$ \\
Erythromycin & $22(91.7)$ & $24(96.0)$ & $17(94.4)$ & $20(95.2)$ \\
Clindamycin & $22(91.7)$ & $24(96.0)$ & $17(94.4)$ & $20(95.2)$ \\
Chloramphenicol & $6(25.0)$ & $3(12.0)$ & $4(22.2)$ & $1(4.7)$ \\
Tetracycline & $22(91.7)$ & $24(96.0)$ & $18(100.0)$ & $20(95.2)$ \\
Trimethoprim & $7(29.2)$ & $7(28.0)$ & $12(66.6)$ & $3(12.0)$ \\
Rifampicin & $2(8.3)$ & $3(12.0)$ & $1(5.5)$ & $0(0.0)$ \\
Fusidic acid & $17(70.8)$ & $24(96.0)$ & $15(83.3)$ & $18(85.7)$ \\
Ciprofloxacin & $18(75.0)$ & $23(92.0)$ & $16(88.8)$ & $21(100.0)$ \\
Mupirocin & $3(12.5)$ & $6(24.0)$ & $12(66.6)$ & $18(85.7)$ \\
Cadmium acetate & $24(100)$ & $24(96.0)$ & $17(94.4)$ & $20(95.2)$ \\
Propamidine isethionate & $23(96.0)$ & $24(96.0)$ & $16(88.8)$ & $19(90.4)$ \\
Ethidium bromide & $23(96.0)$ & $24(96.0)$ & $16(88.8)$ & $19(90.4)$ \\
\hline
\end{tabular}

Figures in parentheses indicate percentages. to gentamicin, kanamycin, streptomycin, erythromycin, clindamycin, tetracycline, trimethoprim, ciprofloxacin, fusidic acid, high-level mupirocin, chloramphenicol, cadmium acetate and propamidine isethionate and ethidium bromide. The prevalence of gentamicin, kanamycin, streptomycin, erythromycin, clindamycin, ciprofloxacin, tetracycline, fusidic acid, cadmium acetate, propamidine isethionate and ethidium bromide resistance remained uniformly high, the already high level mupirocin resistance increased further, over time. However, resistance to chloramphenicol and trimethoprim declined from 25 and $29 \%$ in 1996 to 4.7 and $14.2 \%$ in 2001, respectively. The prevalence of rifampicin resistance remained low, except in 1997. Seven of the 11 OPD isolates were susceptible to gentamicin.

\section{Strain Typing}

The coagulase serotypes of all 88 isolates were determined. Eighty one (92\%) were coagulase serotype 4 . Three isolates, one each from 1996, 1997 and 1998 were nontypeable. Two isolates, both from 1996 were serotype 2, one from 1996 was serotype 6 and one from 1998 was serotype 5 .

\section{Molecular Typing}

The results of molecular typing are summarized in table 2 . The coagulase gene was amplified in all 88 isolates, and the $A l u \mathrm{I}$ restriction endonuclease digestion of the am- plified products yielded four coag-RFLP patterns, designated types $1-4$. These had two AluI restriction fragments but they differed in size (fig. 1). Eighty (91.0\%) of the 88 isolates had the coag-RFLP pattern A. These consisted of all 24 isolates from 1996, 23 of the 25 isolates from 1997, 14 of the 18 isolates from 1998 and 19 of the 21 isolates from 2001. Isolates with coag-RFLP patterns B, C and D occurred sporadically (table 2).

The 88 isolates were classified into seven pulsotypes (types 1-7) according to the criteria proposed for the interpretation of PFGE patterns [15]. The type 1 pulsotype was further divided into eight subtypes, designated types 1a$1 \mathrm{~h}$ (table 2; fig. 2). The type 1 pulsotype was detected in 52 isolates, and the types 1a-1h subtypes, which differed from type 1 pulsotype by $1-5$ fragments, were present in 29 isolates. Thus, in total, the type 1 pulsotype and its subtypes were detected in $81(92.0 \%)$ of the isolates. The remaining six pulsotypes occurred in seven isolates. Analysis of the PFGE patterns of the 11 outpatient isolates revealed that four of them had the type 1 pulsotype, and one isolate each had the type 1a, 1f and $1 \mathrm{~h}$ pulsotypes. The remaining four isolates constituted unrelated PFGE types 3, 5, 6 and 7.

A comparison of the coag-RFLP and PFGE results in table 2 showed that, except for two isolates, isolates with PFGE type 1 pattern and also its subtypes had the coagRFLP type A pattern. The same isolates also belonged to coagulase serotype 4 (not shown). Isolates with PFGE type 2-7 patterns had different coag-RFLP patterns. 


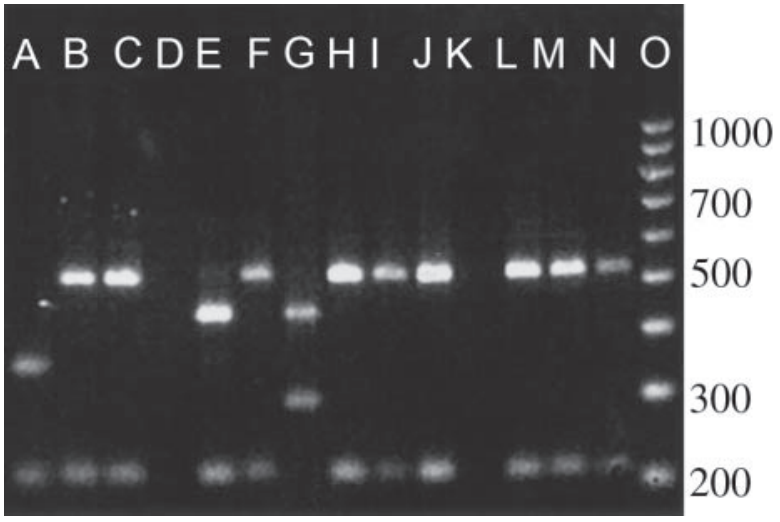

Fig. 1. Coag-RFLP of representative MRSA isolates. Coag-RFLP yielded four patterns (A-D). Lane A, pattern D, sizes are 337 and 200 bp (768 bp). Lanes B, C, F, H, I, J, L, M and N, pattern A (the most common pattern detected in $92.0 \%$ of isolates), sizes are 501 and $200 \mathrm{bp}$ (701 bp). Lane E, pattern C, sizes are 431 and $200 \mathrm{bp}$ (631 bp). Lane G, pattern B, sizes are 431 and 290 bp (721 bp). Lane O, molecular size ladder. Sizes are in kb.

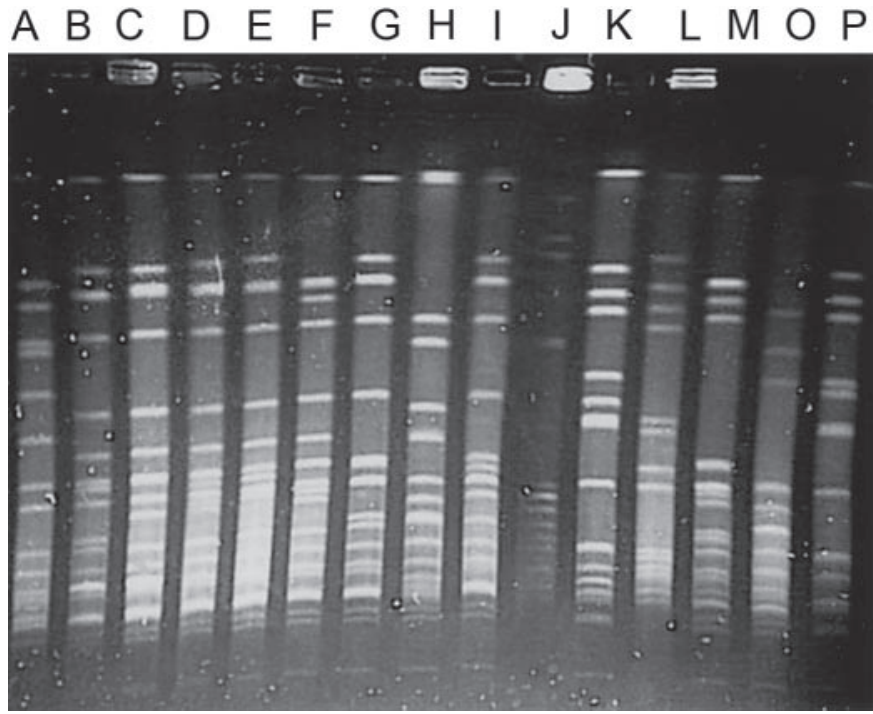

Fig. 2. PFGE types of representative MRSA isolates. Lane A, $S$. aureus strain NCTC8325; lane B, PFGE type 1; lane C, PFGE type 1a; lane D, PFGE type 1b; lane E, PFGE type 1c; lane F, PFGE type 1d; lane G, PFGE type 1e; lane H, PFGE type 1f; lane I, PFGE type 1g; lane J, PFGE type 2; lane K, PFGE type 3; lane L, PFGE type 4; lane M, PFGE type 5; lane N, PFGE type 6; lane O, PFGE type 7 .
Table 2. Comparison of coag-RFLP and PFGE patterns of MRSA isolates

\begin{tabular}{|c|c|c|c|c|c|c|}
\hline \multirow{2}{*}{$\begin{array}{l}\text { PFGE } \\
\text { types }\end{array}$} & \multirow{2}{*}{$\begin{array}{l}\text { Coag-RFLP } \\
\text { patterns }\end{array}$} & \multicolumn{5}{|c|}{ Year of MRSA isolation } \\
\hline & & $\begin{array}{l}1996 \\
(n=24)\end{array}$ & $\begin{array}{l}1997 \\
(n=25)\end{array}$ & $\begin{array}{l}1998 \\
(n=18)\end{array}$ & $\begin{array}{l}2001 \\
(n=21)\end{array}$ & $\begin{array}{l}\text { Total }(\%) \\
(\mathrm{n}=88)\end{array}$ \\
\hline Type 1 & A & 15 & 16 & 7 & 14 & $52(59.0)$ \\
\hline Type 1a & A & 4 & 3 & 2 & 1 & $10(11.3)$ \\
\hline Type $1 b$ & A & - & - & 4 & - & $4(4.5)$ \\
\hline Type 1c & $\mathrm{C}$ & - & - & 2 & - & $2(2.2)$ \\
\hline Type $1 \mathrm{~d}$ & A & 2 & 2 & - & - & $4(4.5)$ \\
\hline Type $1 \mathrm{e}$ & A & - & - & - & 2 & $2(2.2)$ \\
\hline Type $1 f$ & A & 3 & - & - & - & $3(3.4)$ \\
\hline Type $1 \mathrm{~g}$ & A & - & 1 & - & - & $1(1.1)$ \\
\hline Type $1 \mathrm{~h}$ & A & - & 1 & 1 & 1 & $3(3.4)$ \\
\hline Type 2 & $\mathrm{C}$ & - & 1 & - & - & $1(1.1)$ \\
\hline Type 3 & B & - & 1 & 1 & - & $2(2.2)$ \\
\hline Type 4 & $\mathrm{D}$ & - & - & 1 & - & $1(1.1)$ \\
\hline Type 5 & A & - & - & - & 1 & $1(1.1)$ \\
\hline Type 6 & $\mathrm{C}$ & - & - & - & 1 & $1(1.1)$ \\
\hline Type 7 & B & - & - & - & 1 & $1(1.1)$ \\
\hline
\end{tabular}

\section{Genetic Location of Resistance Determinants}

Plasmid analysis revealed that the isolates harbored three to five plasmids per cell with sizes ranging from approximately $2.8 \mathrm{~kb}$ to $38 \mathrm{~kb}$. They all carried a $26-\mathrm{kb}$ and a 3.0-kb plasmid, but they differed in carriage of the 38- and 4.4-kb plasmids (fig. 3). Only the high-level mupirocin-resistant isolates harbored the 38 -kb plasmid. To determine the plasmid resistance phenotypes, four high-level mupirocin resistant and four mupirocin-susceptible isolates, from each year, were selected for curing and 


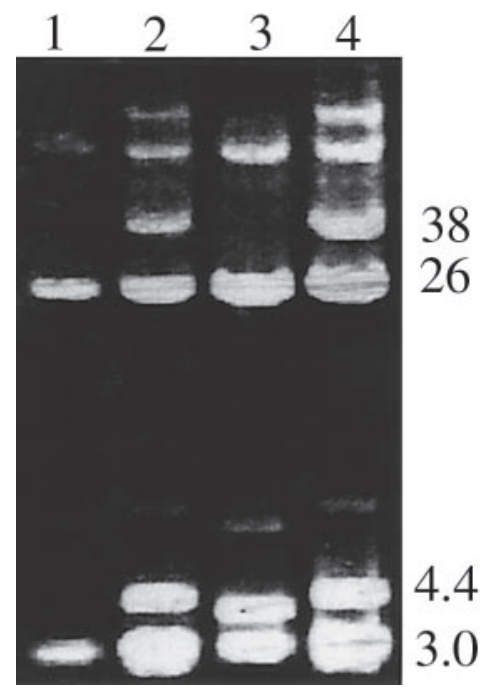

Fig. 3. Plasmid content of representative MRSA isolates. All isolates carried the $26-\mathrm{kb}$ plasmid that encoded cadmium acetate, propamidine isethionate and ethidium bromide resistance. Lanes 1 and 3, mupirocin-susceptible isolates. Lanes 2 and 4, mupirocinresistant isolates containing the $38-\mathrm{kb}$ plasmid that encodes highlevel mupirocin resistance.

transfer experiments. In curing experiments, resistance to cadmium acetate, propamidine isethionate and ethidium bromide were lost simultaneously from all of the isolates and their loss was accompanied by the loss of a $26-\mathrm{kb}$ plasmid. The loss of high-level mupirocin resistance was accompanied by the loss of a $38-\mathrm{kb}$ plasmid and the loss of chloramphenicol resistance was accompanied by the loss of a 4-kb plasmid.

Transfer experiments were conducted to confirm the plasmid resistance phenotypes. In conjugation experiments, using each of the eight isolates as donors, transconjugants were obtained on mupirocin and chloramphenicol selection plates. Transconjugants obtained on mupirocin selection plates were either resistant to mupirocin alone and carried a $38-\mathrm{kb}$ plasmid or resistant to mupirocin and chloramphenicol and carried 38- and 4.4$\mathrm{kb}$ plasmids. Transconjugants obtained on chloramphenicol selection were both resistant to mupirocin and chloramphenicol and carried 38- and 4.4-kb plasmids or were resistant to chloramphenicol alone and contained a 4.4$\mathrm{kb}$ plasmid. Resistance to chloramphenicol, high-level mupirocin, cadmium acetate, propamidine isethionate, and ethidium bromide were transferred in mixed-culture transfer experiments. The transfer of resistance to cadmium, propamidine isethionate, and ethidium bromide was accompanied by the transfer the $26-\mathrm{kb}$ plasmid. Sim- ilarly, the transfer of chloramphenicol and high-level mupirocin resistance was accompanied by the transfer of the 4- and 38-kb plasmids, respectively. Thus, high-level mupirocin resistance was encoded by the $38-\mathrm{kb}$ plasmid, chloramphenicol resistance was encoded by a $4.4-\mathrm{kb}$ plasmid, while the $26-\mathrm{kb}$ plasmid encoded joint resistance to cadmium acetate, propamidine isethionate and ethidium bromide.

\section{Discussion}

This study indicated the presence of antibiotic resistance trends and genetic relatedness of MRSA in a general hospital in Kuwait in 1996-1998 and 2001. MRSA were isolated from all wards except the obstetrics and gynecology ward. The absence of MRSA in the obstetrics and gynecology ward may be attributed to the patient population and their short length of hospital stay. These patients are usually healthy and lack risks factors for MRSA colonization due to prolonged antibiotic therapy and exposure to hospital environment.

Although the isolates were resistant to multiple antimicrobial agents, they were all susceptible to vancomycin, teicoplanin and linezolid, and $93.2 \%$ of them were susceptible to rifampicin. This indicated that these agents could still be used for treating MRSA infections in this hospital. The data demonstrated that while the prevalence of resistance to the aminoglycosides, macrolides, tetracyclines, ciprofloxacin and fusidic acid remained high throughout the study period, already high-level of mupirocin resistance increased steadily over time. Similarly, Al-Haddad et al. [12] reported an increase in highlevel mupirocin resistance from none in 1992, to $56 \%$ in 1997 in the burns unit in Kuwait. Such increases may be attributable to extensive use of antibiotics, which select for the dominance of resistant bacteria [3, 17, 18]. Mupirocin was introduced for clinical use in Kuwait in 1992 and its use contributed to the development of the resistance in the burns unit $[11,12]$. However, no data on mupirocin consumption in the Farwania hospital were available for relating mupirocin utilization with the resistance data obtained, to establish a linkage between mupirocin use and the development of resistance. In contrast, the prevalence of resistance to chloramphenicol and trimethoprim declined. This could be due to the absence of selective pressure since chloramphenicol has not been used in Kuwait since the mid-1980s, and trimethoprim is used sparingly in outpatients for the treatment of nongram-positive infections [Al-Sweih, unpubl. data]. 
Molecular typing of the MRSA isolates by coag-RFLP and PFGE yielded concordant results and allowed the determination of their genetic relatedness. The results showed that the majority had the same coag-RFLP and PFGE patterns (table 2), suggesting that they had a common origin. Isolates with coag-RFLP pattern A were also typed as PFGE pulsotype 1 or its subtypes indicating that an MRSA clone persisted in the hospital for at least 6 years, from 1996 to 2001 . A similar result was reported for MRSA isolated in a burns unit in Kuwait [12]. Krishnan et al. [19] have also reported the persistence of a clone of MRSA in the burns unit in South India for more than 5 years.

The observation that MRSA belonging to PFGE types 2-7 and coag-RFLP genotypes B-D were obtained from outpatients demonstrated the differences between hospital- and community-acquired MRSA. The hospital-acquired MRSA was clonal, persisted for years and spread among different patients. However, few community-acquired isolates occurred sporadically and were heterogeneous. Because of their capacity to spread and persist in the hospital environment, the dominant MRSA clone identified in this study might be considered as epidemic MRSA. Four of the 11 outpatient isolates also had the type 1 PFGE pulsotype that was common among the inpatients. This would suggest the spread of this MRSA clone into the community. It is also possible that these patients had histories of previous hospital admission, which would explain their carriage of the hospital-associated MRSA clone. Different epidemic MRSA strains have been reported from several countries [5-7]. Further studies using multilocus sequence typing $[20,21]$ and the determination of staphylococcus cassette chromosome mec [21] will be needed to determine whether or not the major MRSA clone detected in this study is related to any of the known international epidemic clones.

Curing and transfer experiments demonstrated that the 26-kb plasmid in these isolates coded for resistance to cadmium acetate, propamidine isethionate and ethidium bromide, the 4.4-kb plasmid coded for chloramphenicol resistance and the $38-\mathrm{kb}$ plasmid coded for high-level mupirocin resistance. The 38-kb high-level mupirocin resistance plasmid also appeared in 1996 in the burns unit in Kuwait in 1996 [11, 12]. The detection of the 38-kb high-level mupirocin resistance plasmid in isolates from the general hospital and burns unit in 1996 suggests that it may have been introduced into the two hospitals at about the same time.

\section{Conclusion}

This study demonstrated the presence of two MRSA populations in a general hospital in Kuwait. One type had a capacity to colonize or infect patients in different wards, and persisted in the facilities for at least 6 years. The second type consisted of sporadic isolates that were associated with outpatients and probably represented community MRSA strains.

\section{Acknowledgement}

This study was supported by a Kuwait University Research Administration grant (MI 03/01).

\section{References}

1 Adeyemi-Doro FA, Scheel O, Lyon DJ, Cheng AF: Living with methicillin-resistant Staphylococcus aureus: a 7-year experience with endemic MRSA in a university hospital. Infect Control Hosp Epidemiol 1997; 18:765-767.

-2 Kauffman C, Terpenning M, He X, Zarin L, Ramsey M, Jorgensen K, Sottile W: Attempts to eradicate methicillin-resistant Staphylococcus aureus from a long-term care facility with use of mupirocin ointment. Am J Med 1993; 94:371-378.

-3 Branger C, Gardye C, Lambert-Zechovsky N: Persistence of Staphylococcus aureus strains among cystic fibrosis patients over extended periods. J Med Microbiol 1996;45:294-301.
Roberts RB, De Lencastre H, Eismer W, Severing EP, Shopsin B, Kreiswirth BN, Tomasz A, and the MRSA Collaborative Study Group: Molecular epidemiology of methicillin-resistant Staphylococcus aureus in 12 New York hospitals. J Infect Dis 1998;178:164-171.

-5 Santos Sanches I, Ramirez M, Troni H, Abecassio M, Padua M, Tomasz A, De Lencastre $\mathrm{H}$ : Evidence of the geographic spread of a methicillin-resistant Staphylococcus aureus clone between Portugal and Spain. J Clin Microbiol 1995;33:1243-1246.

-6 De Lencanstre H, Severing E, Milch H, Konkoly Thege M, Tomasz A: Wide geographic distribution of a unique methicillin-resistant Staphylococcus aureus in Hungarian hospitals. Clin Microbiol Infect 1997;3:289-296.

\footnotetext{
Townsend DE, Ashdown N, Bolton S, Bradley J, Duckworth G, Moorhouse EC, Grubb WB: The international spread of methicillin-resistant Staphylococcus aureus. J Hosp Infect 1987;9:60-71.

8 Jarvis WR, Ostrowsky B: Dinosaurs, methicillin-resistant Staphylococcus aureus and infection control personnel: survival through translating science into prevention. Infect Control Hosp Epidemiol 2003;24:392-396.

-9 Richet HM: Better antimicrobial resistance surveillance efforts are needed. ASM News 2001;67:304-309.
} 
10 Udo EE, Al-Obaid IA, Jacob LE, Chugh TD: Molecular characterization of epidemic ciprofloxacin- and methicillin-resistant Staphylococcus aureus strains colonizing patients in an intensive care unit. J Clin Microbiol 1996;34: 3242-3244.

11 Udo EE, Farook VS, Mokadas EM, Jacob LE, Sanyal SC: Molecular fingerprinting of mupirocin-resistant methicillin-resistant Staphylococcus aureus from a burn unit. Int J Infect Dis 1999;3:82-87.

12 Al-Haddad AM, Udo EE, Mokadas EM, Sanyal SC, Grubb WB: Persistence of a clone of methicillin-resistant Staphylococcus aureus in a burns unit. J Med Microbiol 2001;50:558564.

13 National Committee for Clinical Laboratory Standards. Performance for Disk Diffusion Susceptibility Tests. Approved Standards. Wayne, National Committee for Clinical Laboratory Standards, 2000.
14 Aarestrup FM, Dangler CA, Sordillo LM: Prevalence of coagulase gene polymorphism in Staphylococcus aureus isolates causing bovine mastitis. Can J Vet Res 1995;59:124-128.

15 Tenover FC, Arbeit RD, Goering RV, Mickelson PA, Murray BE, Persing DH, Swaminathan B: Interpreting chromosomal DNA restriction patterns produced by pulsed-field gel electrophoresis: criteria for bacteria strain typing. J Clin Microbiol 1995;33:2233-2239.

16 Udo EE, Jacob LE: Conjugative transfer of high-level mupirocin resistance and the mobilization of non-conjugative plasmids in Staphylococcus aureus. Microb Drug Resist 1998;4: 185-193.

17 Chang SC, Chang HJ, Hsiao M: Antibiotic usage in public hospitals in Taiwan. J Microbiol Immunol Infect 1998;31:125-132.
18 McDonald LC, Yu HT, Yin HC, Hsiung AC, Ho M, and Antibiotic Use Working Group: Use and abuse of surgical antibiotic prophylaxis in hospitals in Taiwan. J Formos Med Assoc 2001;100:5-13.

19 Krishnan PU, Mahalakshmi P, Shetty N: Strain relatedness of endemic MRSA isolates in a burns unit in South India - a five-year study. J Hosp Infect 2002;52:181-184.

20 Enright MC, Day NPJ, Davies CE, Peacock SJ, Spratt BG: Multilocus sequence typing for characterization of methicillin-resistant and methicillin-susceptible clones of Staphylococcus aureus. J Clin Microbiol 2000;38:10081015.

21 Melter O, Aires de Sousa M, Laskafeldova K, Urbaskova P, Wunschova M, De Lencastre H: Delineation of the endemic and sporadic methicillin-resistant Staphylococcus aureus clones in a Czech hospital. Microb Drug Resist 2004; 10:218-223. 